\title{
One-step grown aligned bulk carbon nanotubes by chloride mediated chemical vapor deposition
}

\author{
Yoku Inoue, ${ }^{1, a}$ Kazuyuki Kakihata, ${ }^{1}$ Yusaku Hirono, ${ }^{1}$ Toshinori Horie, ${ }^{1}$ Akihiro Ishida, ${ }^{1}$ \\ and Hidenori Mimura ${ }^{2}$ \\ ${ }^{1}$ Department of Electrical and Electronic Engineering, Shizuoka University, 3-5-1 Johoku, \\ Hamamatsu 432-8561, Japan \\ ${ }^{2}$ Research Institute of Electronics, Shizuoka University, 3-5-1 Johoku, Hamamatsu 432-8011, Japan
}

(Received 1 February 2008; accepted 19 April 2008; published online 29 May 2008)

\begin{abstract}
We found the easy and efficient synthesis method of the vertically aligned ultralong multiwalled nanotubes using iron chloride powder. The 2.1-mm-long bulk nanotubes can be grown by conventional thermal chemical vapor deposition on bare quartz surface with the single gas flow of acetylene for $20 \mathrm{~min}$. In addition to the high growth rate, the bulk of carbon nanotubes is easily spun into the yarn by pulling it out, and the present method also provides the coating ability with nanotubes as a new functionality of this nanomaterial. (C) 2008 American Institute of Physics.
\end{abstract} [DOI: $10.1063 / 1.2937082$ ]

Since their discovery in $1991,{ }^{1}$ carbon nanotubes (CNTs) have been regarded as a key nanomaterial for a wide range of applications. ${ }^{2}$ Their small tubular structure is responsible for diverse features, such as high mechanical strength, ${ }^{3}$ strong electric properties, ${ }^{4}$ good heat conductance, ${ }^{5}$ and high electron emission, ${ }^{6}$ which are of interest for academic researchers and industries. ${ }^{7,8}$ However, despite several efforts, ${ }^{9-13}$ a suitable mass-synthesis method for CNTs that promotes widespread practical applications is yet to be established.

Here, we present a simple and easy way for mass synthesis that yields ultralong, vertically aligned multiwalled CNTs (MWCNTs). This method requires no additional process for catalyst preparation (predeposition) and only requires iron chloride powder and acetylene gas used. We found that high dehydrogenation activity of iron chloride on acetylene increases the growth rate of CNTs compared to conventional predeposited metal catalysts. The lengths of obtained MWCNTs ranged up to the millimeter scale, and they can easily be spun into yarn by hand with the naked eyes. In addition, this method can be used to coat the entire surface of a target with MWCNT using iron chloride in vapor phase as a growth initiator. The most attractive feature of the proposed method is that it is extremely simple, and therefore, could be used in any laboratory for bulk production of MWCNTs.

Vertically aligned CNTs (VACNTs) were synthesized using a conventional thermal chemical vapor deposition (CVD) system. A smooth quartz substrate was placed at a center of horizontal quartz tube furnace $(40 \mathrm{~mm}$ in diameter and $30 \mathrm{~cm}$ in length) with iron chloride $\left(\mathrm{FeCl}_{2}\right)$ powder $(99.9 \%$, Kojundokagaku Laboratory) using a quartz boat. As a pretreatment, the quartz substrate was cleaned using ethanol. Typically, a thin metallic film deposited on a substrate is widely used as a catalyst; however, in the proposed method, such a film need not be predeposited. During heating, the sample was maintained at vacuum of $1 \times 10^{-3}$ Torr, and once the optimal growth temperature was reached, it was purged with acetylene (98\%, Japan Air Gases) gas using a mass flow controller. CVD growth was carried out at a furnace tempera-

${ }^{a)}$ Electronic mail: tyinoue@ipc.shizuoka.ac.jp. ture of $820^{\circ} \mathrm{C}$ at 10 Torr. Hereafter, we refer to the proposed method as "chloride mediated CVD" (CM-CVD). Features such as using a pure gas flow and not using a metallic film on the substrate are peculiar to CM-CVD and could lead to significant reduction in the mass-production costs of CNTs.

A VACNT sample is shown in Fig. 1. Densely grown CNTs are vertically aligned on a quartz substrate (10 $\times 10 \mathrm{~mm}^{2}$ ). The top (grown) surface is macroscopically flat, i.e., the CNT length is uniform throughout the substrate [Fig. 1(a)]. Figure 1(b) compares the samples before and after the growth. The left and right images show a corner of the quartz substrate before and after the growth, respectively. From Figs. 1(a) and 1(b), one can observe the vertically aligned growth throughout the surface, including the side and back surfaces. Figure 1(c) shows a side-view image of VACNTs grown for $20 \mathrm{~min}$ using a scanning electron microscope (SEM). Vertical alignment is again clearly confirmed from the image. The length of the VACNTs is $2.1 \mathrm{~mm}$. This growth rate of $2.1 \mathrm{~mm} / 20 \mathrm{~min}$ is remarkably high. ${ }^{11,13} \mathrm{Al}$ though VACNTs seem to be macroscopically straight, they are found to be partially curved, as shown in Fig. 1(d). The obtained CNTs were confirmed to contain very few Fe particles, and to be multiwalled from transmission electron microscopy (TEM) image shown in Fig. 1(e). The diameter is $30-50 \mathrm{~nm}$.

In CM-CVD, the stability of the VACNTs grown on a substrate depends on the growth conditions, such as the growth time and pressure. It is found that the 2.1-mm-long CNTs adhere strongly to the substrate, while the VACNTs grown under 4 Torr detached easily, leaving a very clean surface. For this type of VACNTs, one can easily spin a CNT yarn using tweezers or an adhesive tape. ${ }^{14}$ As seen in Fig. 2(a), we obtained a $15.5 \mathrm{~cm}$ CNT yarn by pinching and pulling out CNTs. ${ }^{15}$ Figure 2(b) shows that during the pulling, a tensioned yarn extended up to $20 \mathrm{~cm}$ in length.

We got the idea of the CNT-coating growth from the entire surfaces growth. To demonstrate the CNT-coating ability, the CNT growth was first examined on a quartz tube with a $6 \mathrm{~mm}$ diameter [Fig. 3(a)]. The CNTs were grown throughout the outer and inner surfaces. The magnified image clearly 

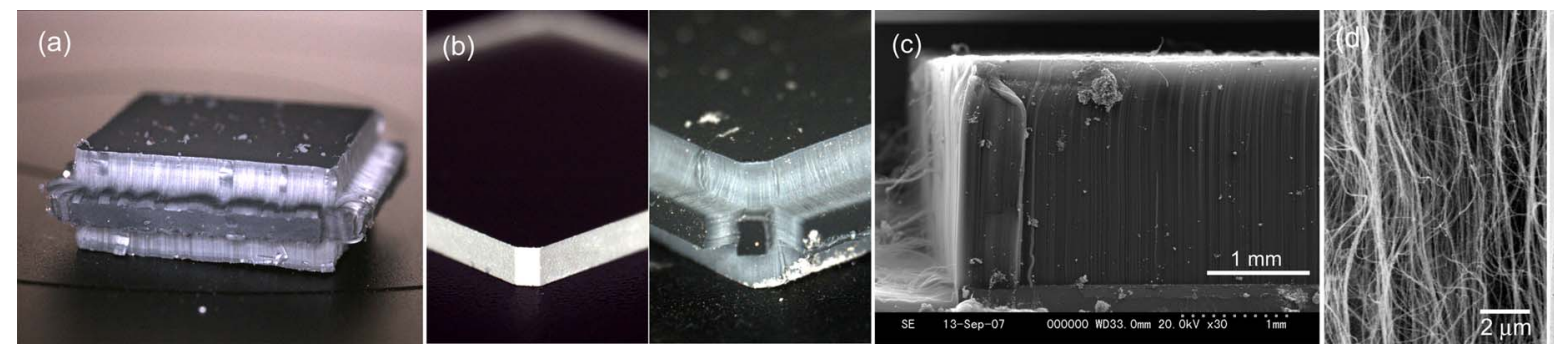

(e)

FIG. 1. (Color online) Multiview images of the sample. (a) VACNTs were grown not only on the top surface but also on the side and back surfaces. (b) Growth morphology of a corner of the sample. Since the corner of the quartz substrate was cut (left image), VACNTs at the corner were grown separately. (c) SEM image of 2.1-mm-long VACNTs. (d) Magnified image revealed the wavy morphology of the CNT. (e) TEM image of the VACNTs. Inset shows a high resolution image of multiwalled CNT (scale bar $=10 \mathrm{~nm})$.

shows the vertical alignment of the CNTs on the surfaces. This result indicates that CM-CVD can be used for coating CNTs on both inner and outer surfaces. We further examined this coating ability using quartz fiber wool of $3-5 \mu \mathrm{m}$ diameter, which is a more complicated coating target, as shown in Fig. 3(b). After the coating process, the quartz wool appeared black [Fig. 3(c) inset]. SEM observation revealed that the individual quartz fibers were completely coated with hairlike CNTs, as shown in Fig. 3(c). The fibers increased to $30-50 \mu \mathrm{m}$ in diameter due to the coating. Figure 3(d) shows the results of another CNT coating using $\mathrm{FeCl}_{3}$ powder, and a different surface morphology was observed. CNTs were firmly coated on the fibers, and it was interesting to see that some even grew along the length of the fibers. At present, this morphological change is not completely understood. It should be noted that the macroscopic coating morphology extended homogeneously from the surface to the inside of a quartz wool ball.

The advantage of applying this technique is that it can easily be used to coat structurally complex samples. Achieving this using the conventional CVD method is far from trivial because that would require overcoming the difficulty of achieving a predeposition of a metallic film over rough, indented, tubular porous structures.

In this study, we propose the CNT growth model in CMCVD. To understand the reaction processes, the transition of the partial pressure of released gases was measured using a quadrupole mass analyzer (MKS instruments, PPT-C200F2T), as shown in Fig. 4. In the mass analysis, a part of the released gas was sampled, and the operating pressure was
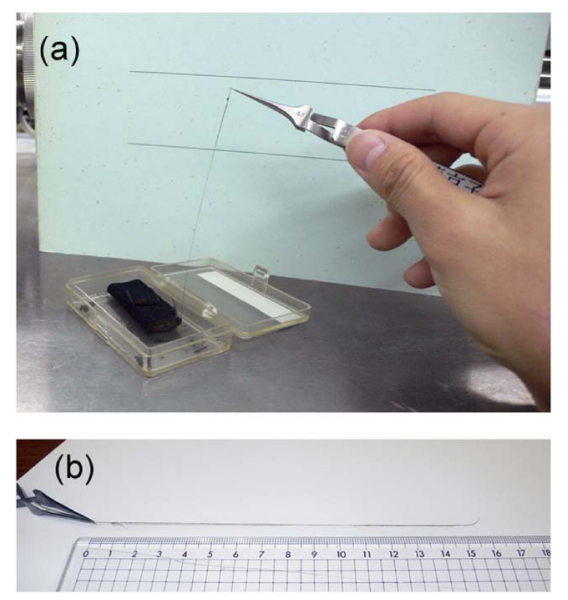

FIG. 2. (Color online) Spinning CNTs. (a) CNTs yarn is being spun by pulling them out from the VACNTs sample using tweezers. (b) Spun yarn with a length of $15.5 \mathrm{~cm}$. pressed as follows:
Downloaded $16 \mathrm{Jul} 2008$ to 133.70 .80 .50 . Redistribution subject to AlP license or copyright reduced to $1 \times 10^{-5}$ Torr. After the beginning of the growth process with the acetylene $\left(\mathrm{C}_{2} \mathrm{H}_{2}, 26 \mathrm{amu}\right)$ feed, $\mathrm{HCl}$ (36 amu) was generated for a very short time as a trigger signal. After some time, hydrogen (2 amu) increased for $5 \mathrm{~min}$, and then decreased gradually. We believe that the generation of the two gases $\left(\mathrm{HCl}\right.$ and $\left.\mathrm{H}_{2}\right)$ relates to "bud beak" and "CNT growth," respectively.

$\mathrm{HCl}$ generation can be expressed as

$$
\mathrm{FeCl}_{2}+\mathrm{C}_{2} \mathrm{H}_{2} \rightarrow \mathrm{FeC}_{2}+2 \mathrm{HCl} \text {. }
$$

Since $\mathrm{FeCl}_{2}$ is completely vaporized at $820{ }^{\circ} \mathrm{C},{ }^{16}$ this reaction mainly occurs in the vapor phase, and therefore, $\mathrm{FeC}_{2}$ molecules or related carbon-rich iron carbide clusters may first form. Due to the low vapor pressure of iron carbide, those carbon-rich iron carbide species are considered to nucleate into nanoparticles as a result of multiple collisions, and to be deposited on the surfaces in the heated zone. Then, the $\mathrm{FeC}_{2}$ nanoparticles segregate into graphene layers as follows: ${ }^{17}$

$$
\mathrm{FeC}_{2} \rightarrow \mathrm{FeC}_{2-x}+x \mathrm{C} \text {. }
$$

We believe that CNT growth in CM-CVD is initiated during the segregation of the graphene layers from carbon-rich iron carbide $\left(\mathrm{FeC}_{2}\right)$ as predicted by Jourdain et al. ${ }^{18}$ Once budlike CNT structures are formed, the growth is triggered.

The subsequent hydrogen generation reflects the carbon supply for the growth of the CNTs because hydrogen generation is the result of only the decomposition of acetylene. There are two possible reactions that result in the decomposition into hydrogen. One is a Fe-catalyzed dehydrogenation reaction, and the other is the reaction mediated by iron chloride. However, from our conventional CVD experiments, we found that small amounts of hydrogen are generated during the growth on a Fe predeposited quartz substrate, and the hydrogen generation is terminated within $3 \mathrm{~min}$. In addition, the length of the CNTs grown using this simple thermal CVD method was less than $300 \mu \mathrm{m}$. Therefore, these results indicate that the observed high-speed growth is mediated by iron chloride. At first, iron chloride surfaces may appear on the catalyst nanoparticles at the root of CNTs due to the adsorption of the remaining $\mathrm{FeCl}_{2}$ and/or chlorination of surface $\mathrm{Fe}$ atoms with the remaining $\mathrm{HCl}$, according to the following well-known reaction:

$$
\mathrm{Fe}+2 \mathrm{HCl} \rightarrow \mathrm{FeCl}_{2}+\mathrm{H}_{2} .
$$

Then, acetylene is highly decomposed at the root of CNTs due to the high dehydrogenation activity of iron chloride. ${ }^{19}$ Reactions of acetylene at the iron chloride surfaces are ex- 

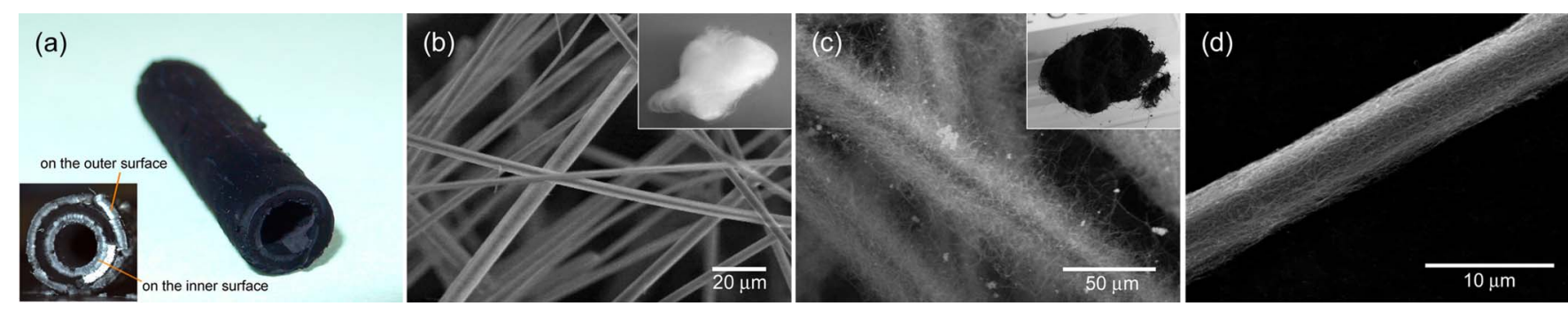

FIG. 3. (Color online) Growth of CNT coating. (a) Coating on a quartz tube with $6 \mathrm{~mm}$ diameter. VACNTs were grown not only on the outer surfaces but also on the inner surface. (b) SEM image of quartz wool fibers used for the CNT growth. Inset shows a photograph of wool ball. (c) SEM image of quartz fibers coated with hairlike CNTs grown with $\mathrm{FeCl}_{2}$. Inset shows a photograph of CNT-coated quartz wool. (d) SEM image of a quartz fiber tightly coated with CNTs grown with $\mathrm{FeCl}_{3}$.

$$
\mathrm{FeCl}_{2}+\mathrm{C}_{2} \mathrm{H}_{2} \rightarrow \mathrm{FeC}_{2}+2 \mathrm{HCl}
$$

In the reaction, acetylene is decomposed, and $\mathrm{FeC}_{2}$ phase is again considered to form. Since $\mathrm{FeC}_{2}$ highly exceeds a solubility limit of carbon in iron, carbon is expected to rapidly segregate as follows:

$$
\mathrm{FeC}_{2} \rightarrow \mathrm{FeC}_{2-x}+x \mathrm{C} \text {. }
$$

By this process, the CNTs grow because of this carbon segregation. Here, Eq. (5) is the same with Eq. (2). This reaction cycle is expected to repeat rapidly, which means the ultralong VACNTs are grown in a short amount of time. This reaction cycle, where iron chloride acts as catalyst, is one of the most important discoveries of this study.

For the CNT coating, a vapor phase reaction of iron chloride with acetylene is essential. By observing the heating process through a viewing port, we found that $\mathrm{FeCl}_{2}$ started to vaporize at $550{ }^{\circ} \mathrm{C}$, and completely vaporized over $800{ }^{\circ} \mathrm{C}$, when the local vapor pressure reached 70 Torr. During this heating process, $\mathrm{FeCl}_{2}$ vapor spreads across the entire heated space. Since acetylene flow causes the sublimation of $\mathrm{FeC}_{2}$ nanoparticles on the entire surfaces, CNTs can be grown everywhere, even on micron-spaced quartz wool. This growth initiation via vapor phase $\mathrm{FeCl}_{2}$ is another important finding of this study.

In summary, we established a simple one-step growth method of aligned bulk CNTs mediated by iron chloride. The CM-CVD offers a potentially viable VACNT mass production method. In the present system, we can grow $1 \mathrm{~g}$ of VACNT for a $2 \mathrm{~h}$ process time including $20 \mathrm{~min}$ of growth time, and material cost performance is as low as US $\$ 0.5 / \mathrm{g}$. If a large number of flat plates are arranged in a growth

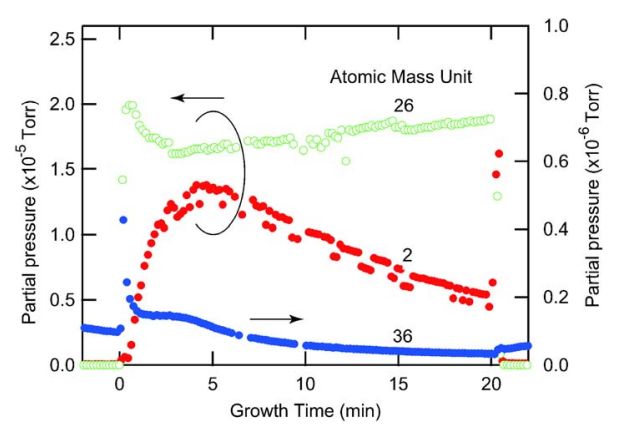

FIG. 4. (Color online) Sampled partial pressure of released gases as a function of growth time. Numbers denote the atomic mass unit, where 2, 26, and 36 are the atomic mass units of hydrogen (left axis), acetylene (left), and hydrogen chloride (right), respectively. chamber, then a significant amount of VACNTs can be easily obtained from the entire surface by the high-speed coating growth. Although our VACNTs length has not exceeded the world record ${ }^{20}(18 \mathrm{~mm}$ : reported by University of Cincinnati researchers), the rate of "production per process time and cost $[g / h-\$]$ " might be significantly high. We believe that this very simple one-step growth technology, based on bifunctionality of $\mathrm{FeCl}_{2}$, will contribute to the development of new CNT applications.

We thank Professor Hironobu Umemoto for fruitful discussion. This study was partly supported by a Grant-in-Aid of Japan Society for the Promotion of Science, The Asahi Glass Foundation, and Research Foundation for OptoScience and Technology.

${ }^{1}$ S. Iijima, Nature (London) 354, 56 (1991).

${ }^{2}$ R. H. Baughman, A. A. Zakhidov, and W. de Heer, Science 297, 787 (2002).

${ }^{3}$ M. M. J. Treacy, T. W. Ebbesen, and J. M. Gibson, Nature (London) 381, 678 (1996).

${ }^{4}$ T. W. Ebbesen, H. J. Lezec, H. Hiura, J. W. Bennett, H. F. Ghaemi, and T. Thio, Nature (London) 382, 54 (1996).

${ }^{5}$ P. Kim, L. Shi, A. Majumdar, and P. L. McEuen, Phys. Rev. Lett. 87, 215502 (2001)

${ }^{6}$ W. A. de Heer, A. Châtelain, and D. Ugarte, Science 270, 1179 (1995).

${ }^{7}$ N. S. Lee, D. S. Chung, I. T. Han, J. H. Kang, Y. S. Choi, H. Y. Kim, S. H. Park, Y. W. Jin, W. K. Yi, M. J. Yun, J. E. Jung, C. J. Lee, J. H. You, S. H. Jo, C. G. Lee, and J. M. Kim, Diamond Relat. Mater. 10, 265 (2001).

${ }^{8}$ Y. Saito and S. Uemura, Carbon 38, 169 (2000).

${ }^{9}$ H. M. Christen, A. A. Puretzky, H. Cui, K. Belay, P. H. Fleming, D. B. Geohegan, and D. H. Lowndes, Nano Lett. 4, 1939 (2004).

${ }^{10}$ G. Eres, A. A. Puretzky, D. B. Geohegan, and H. Cui, Appl. Phys. Lett. 84, 1759 (2004)

${ }^{11}$ K. Hata, D. N. Futaba, K. Mizuno, T. Namai, M. Yumura, and S. Iijima, Science 306, 1362 (2004).

${ }^{12}$ A. J. Hart and A. H. Slocum, J. Phys. Chem. B 110, 8250 (2006).

${ }^{13}$ S. Chakrabarti, T. Nagasaka, Y. Yoshikawa, L. Pan, and Y. Nakayama, Jpn. J. Appl. Phys., Part 2 45, L720 (2006).

${ }^{14}$ K. Jiang, Q. Li, and S. Fan, Nature (London) 419, 801 (2002).

${ }^{15}$ See EPAPS Document No. E-APPLAB-92-084821 for CNT yarn being spun by pinching and pulling out from the VACANT sample using tweezers. For more information on EPAPS, see http://www.aip.org/pubservs/ epaps/html.

${ }^{16}$ CRC Handbook of Chemistry and Physics, 84th ed., edited by D. R. Lide (CRC, Boca Raton, 2003).

${ }^{17}$ K. Kosugi, M. J. Bushiri, and N. Nishi, Appl. Phys. Lett. 84, 1753 (2004).

${ }^{18}$ V. Jourdain, H. Kanzow, M. Castignolles, A. Loiseau, and P. Bernier, Chem. Phys. Lett. 364, 27 (2002).

${ }^{19}$ T. Laude, H. Kuwahara, and K. Sato, Chem. Phys. Lett. 434, 78 (2007).

${ }^{20} 18$-mm-long VACNTs was issued by press release from National Science Foundation (http://www.nsf.gov/news/news_summ.jsp?cntn_id $=108992 \&$ org $=$ NSF). 